\title{
THE PROBABILITY OF UNCERTAINTY: ROMANIA'S GROWTH PERSPECTIVES
}

\section{Ionuţ Gavriş, Valentin Toader}

Babes-Bolyai University, Faculty of Business, Cluj-Napoca, Romania

ilgavris97@gmail.com

valentin.toader@ubbcluj.ro

\begin{abstract}
This paper follows the already existing literature to address three main pressing concerns that Romania is currently facing with respect to sustainable development and economic growth, which has likely been impeded as a direct consequence of the ongoing sanitary crisis. The research questions of this paper were asked with the consideration of finding possible solutions to the current developments. These questions were "How will the global health crisis project on Romania's GDP growth in 2021 and going forward?", "To what extent can Romania face its current challenges with respect to the budgetary pressures?". The research itself was conducted using the $R$ programming language with quantitative data, namely quarterly GDP starting from 2005 Q1 onwards and its main objective is to simulate Romania's GDP to answer the aforementioned questions. Therefore, this research is empirical in the sense that it derives the simulated data from real data which was taken from Eurostat. The main findings of this research were that Romania's GDP could grow by a mean $3.2 \%$ in 2021, which is more conservative than the existing institutional forecasts made by the European Commission, the International Monetary Fund, World Bank and the Romanian National Commission for Strategy and Forecasting, implying that the majority of the simulated outcomes have been in that respective range of values. Moreover, this research also finds that consumption is expected to increase by approximately $4.2 \%$ and that the budget deficit relative to GDP could go as low as to negative $7.8 \%$. This paper creates value for future policymaking as it proposes solutions which could help Romania going forward. Thus, this paper creates value for not only the policymakers but the society as a whole.
\end{abstract}

Keywords: GDP; probability; distribution; growth; forecasting; Romania;

JEL Classification: C25; C53; E17

\section{Introduction}

It is undoubtedly that the ongoing sanitary crisis has affected both the economy and society as a whole in 2020. Having this in mind, a few questions, which can help in reducing uncertainty, remain unanswered. It is unclear how the current crisis can project its effects on Romania's economic development in 2021 and going forward, and to what extent could the country face its challenges concerning the budgetary pressures. This paper attempts to answer these questions by running probability distribution simulations using quarterly data and multiple models. Moreover, possible solutions that may aid in policymaking are proposed at the end of this page. The purpose of these proposals is to pave the way for further research, as this paper does not analyze the potential impact of the respective, it addresses some of the most pressing concerns the country is currently facing. The rest of this paper is organized as follows; section one shows the already existing literature on the topic, section two describes the models used, how they were coded and what programming language was utilized together with the research questions, section three explains how the models were tested and the main findings, and section four ends the paper with a discussion, a conclusion and the research limitations. The references section follows right after. 


\section{Literature Review}

If a country's Gross Domestic Product (GDP) were to be a deterministic process, then the following year's GDP could only have one possible outcome since GDP would be solely determined by the parameters that condition the outcome, that is, implying that no randomness occurs over time, and therefore the output would always be the same. On this basis, going by the expenditure approach, where GDP is computed as the sum of consumption (C), investment (I), government expenditure $(G)$, and net exports (E-I), all variables would be known beforehand and no changes would ever be made. (Landefeld, 2008)

However, this is seldom the case since there are events that can significantly disrupt economic growth. As such, a country's GDP over time is a stochastic process since there are multiple possibilities that can occur given a set of predefined events. Because the parameters that can affect GDP cannot be fully encompassed in a one state model, GDP can be viewed as a Markov Chain. Metropolis and his peers (1953) were the first to consider this method, and later on, Hastings (1970) developed an algorithm that made the simulation of stochastic processes possible. Geman and Geman (1984) further developed this method through a Gibbs sampling. Tanner and Wong (1987) extend this work by showing how posterior distributions can be computed whenever data augmentation can be generated. They conclude that if the sample size is large in each simulation, their algorithm can be interpreted as a method of solving a fixed-point problem.

On the other hand, Little (2006) explains how inferences in a statistical model should be Bayesian with a frequentist approach while Kruger and his peers (2017) find, based on frequentist principles, that the kernel density estimator, which is a method to estimate the probability density, performs poorly for small sample sizes and the continuously ranked probability score is dominated by the empirical cumulative distribution. They also argue that Gaussian approximations generally fail to be consistent. Wolf and his peers (2017) consider three MCMC approaches, namely, Metropolis with Gibbs sampling, Slice with Gibbs sampling and Hamiltonian Monte Carlo. They note how the latter two models are not widely used in Bayesian spatial econometrics and assess all three methods in four different scenarios, focusing on algorithms that sample from the posterior distribution. They find that standard simulation packages require significant adaptations to be viable, suggesting customization is needed to attain unbiased results since the effective sample size is often smaller than the nominal sample size of the autoregressive spatial parameter, and stopping the simulation early may result in understated posterior credible intervals.

Glocker and Wegmuller (2017) study the business cycles in the Swiss economy using a dynamic factor Markov-switching model. They argue that the proposed model demonstrates high accuracy forecasts and that it accurately coincides with the Swiss cycle fluctuations, including the 2008-09 financial crisis.

Roy (2020) addresses two issues regarding the implementation of such simulations, where to start and stop the algorithm, as these two factors relate to the convergence between the Markov Chain and the stationarity and convergence of Monte Carlo estimations. He argues that the algorithm should be started in high density areas, but only after enough iterations have been computed so as to find the appropriate area. He also explains how stopping the simulation early leads to inaccurate inferences and so, the simulation should be stopped only after the real valued function converges the particular feature of the target density. Moreover, he suggests that diagnostic tools are a fundamental requirement for deciding the degree of stationarity and convergence, but in general, the longer the chain is run, the better the output.

In terms of applying these methods on the Romanian economy, several search terms such as "probability distribution Romania", "Markov Chains Romania", "Metropolis sampling Romania", "Markov Chain Monte Carlo Romania" and others have been tried, with the filter 
for articles no older than 2015. Even though the search has yielded plenty of literature, there have not been many that focus on economics and forecasting. Particularly, Kluza (2016) focuses on simulations regarding the sustainability of the government debt levels. He finds that some scenarios indicate a high vulnerability of the local governments to changes in the interest rates. Savoiu and his peers use the method to simulate the value of European convergence funds that could be absorbed by Romania during 2014-2020 while Simionescu (2020) focuses her attention on Bayesian forecasts with Monte Carlo simulations to improve inflation. As a result, the search yielded no results that incorporate simulations that primarily focus on Romania's GDP going forward in literature from 2015 onwards, and especially, no results were yielded that incorporate the possible outcomes of the lockdowns. Therefore, it is imperative to consider these developments and simulate Romania's probability distribution of economic development.

\section{Methodology}

This research uses quantitative data on the assumption that a qualitative method such as a focus group would not be representative for an entire economy. Moreover, this paper makes use of Romanian quarterly GDP (and aggregates) data from 2005 Q1 onwards. The paper uses secondary data, which was collected from Eurostat, and all the data points are expressed in EUR as a currency. 2005 was chosen as a reference point since it was that year when the country chose inflation targeting regarding its monetary policy (Daianu \& Kallai, 2008). Among the existing literature, Svensson (2010) sees this approach as a successful way of stabilizing inflation and the real economy while also providing a high degree of transparency.

Multiple problems have been identified which makes this research necessary. Even though there have been studies that focus on forecasting Romania's economic growth through Bayesian methods or even through Monte Carlo simulations for inflation (Simionescu, 2020), little to no literature is available concerning estimating GDP through Markov Chains or Monte Carlo or any other probability distribution approach.

Due to the high number of variables that can affect economic growth, one single forecast cannot be said to provide accurate results. Thus, in a probability distribution, more simulations would indicate far more accurate results than one single forecast. For example, Lerche and Mudford (2005) explain the tradeoff between the number of simulations and the time it takes to provide accurate results. A standard procedure is to run a fixed number of simulations and to evaluate the mean and the standard error. Then, to increase the number of simulations and re-evaluate the aforementioned. They point out that enough simulations should be run up to the point where one determines that the change in the mean with the increase in number of runs is less than the pre-specified degree of accuracy. As mentioned, this trial and error approach comes at the expense of time needed to converge with the predefined degree of accuracy. Therefore, for the purpose of this paper, at least one million simulations are run for each model, and the results are compared to the already existing institutional forecasts.

Another consideration is the effect of the global health crisis. It is yet to be determined how the economic landscape was reshaped since some economies are going back in lockdowns. As such, little literature is available on these topics. This paper can contribute to elucidating the question of what the full effects could be. Moreover, Romania is faced with a high degree of uncertainty with regards to its current economic system, that is, a declining population (World Bank), a high amount of economic stress due to its budgetary spending, implying a 7.16\% deficit in 2021 and $5.84 \%$ in 2022 according to Bloomberg, and a low digitalization rate compared to the EU average (European Commission). This paper looks at different scenarios, attempting to resolve these issues, that could later be used in future policy making. 
Two research questions have been asked to find a solution to these prevalent issues. They are listed below:

- How will the global health crisis project on Romania's GDP growth in 2021 and going forward?

- To what extent can Romania face its current challenges concerning increased governmental expenditure, output levels? And what are the possible solutions to ensuring a sustainable system going forward?

Probability distributions with respect to Romania's economic development are a necessity when considering these two questions since it implies that policymakers and other stakeholders consider multiple possible outcomes to ensure things do not take a turn for the worse. In order to answer these questions and find solutions to the stated issues, one software package was used, namely, the programming language $R$ to create custom simulations specifically tailored for Romania.

\section{The Distribution}

According to Olerichs (2017), a solution for simulating future economic growth is to estimate the probability distribution of possible economic outlooks using the variance of growth. This paper follows the aforementioned approach in the sense that it uses the same distribution selection procedure and Bayesian inferences.

A proper distribution must be chosen to produce unbiased results. Thus, by examining the historical quarterly change in Romania's GDP, one can determine what a distribution could look like for this paper.

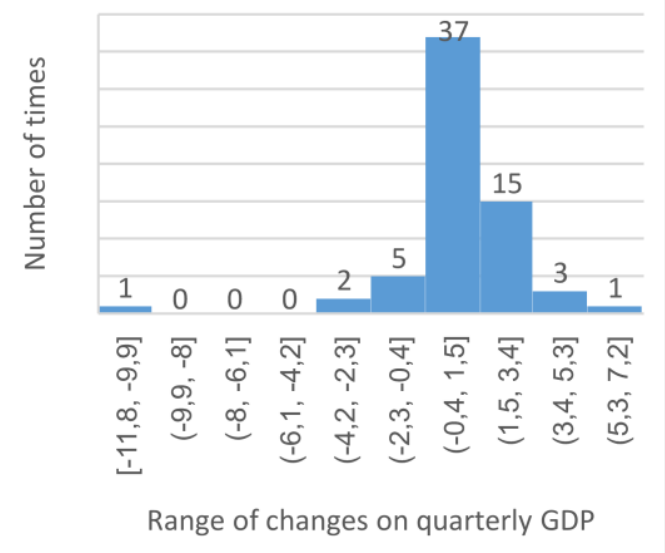

Figure 1: Romania's quarterly GDP change Source: Eurostat

Therefore, following the existing literature and considering the infinite number of possibilities, it has been decided that Romania's quarterly fractional change in GDP fits an asymmetric Student's t distribution.

\subsection{The Historical Model}

This model is the foundation on which all other models in this paper have been simulated. In this particular instance, a normal distribution was fit for the simulated cases. As Olerichs (2017) points out, this method is conservative in the sense that it does not account for the potential decrease in relative fractional growth as a country develops and thus, growth would be somewhat constant over time. 


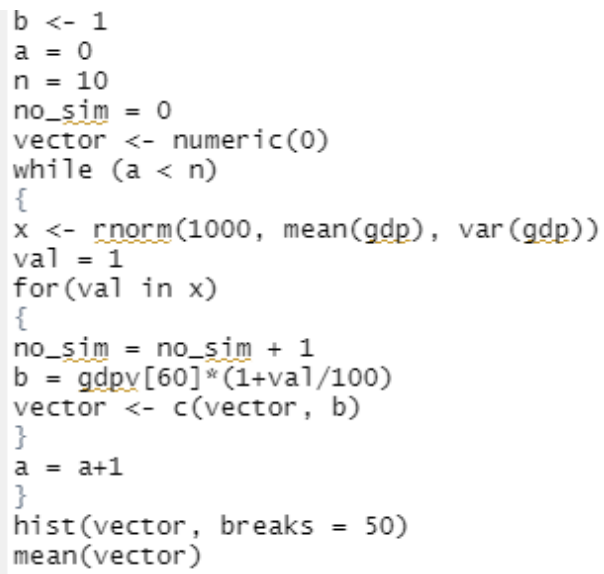

Figure 2: The Historical Model

Source: Own code

The lines of code above simulate 100 cases for each "a" until "a" is equal to " $n$ " and the distribution is fit from a different sample each time to avoid a single sample bias. Thus, for "n=100", 100 different distributions are sampled, each containing 100 possible outcomes. In order to create unbiased results, the simulated distribution follows the mean of the historical GDP as well as its' variance.

\subsection{The Gross-Value Added Model}

This model follows the same coding logic as the historical model, albeit with some differences. First, the total sectorial output refers to the Gross Value Added (GVA) by sector as detailed by Eurostat (2021). Second, GDP is estimated by summing all sectors and each sector is estimated using a similar code to the historical model.

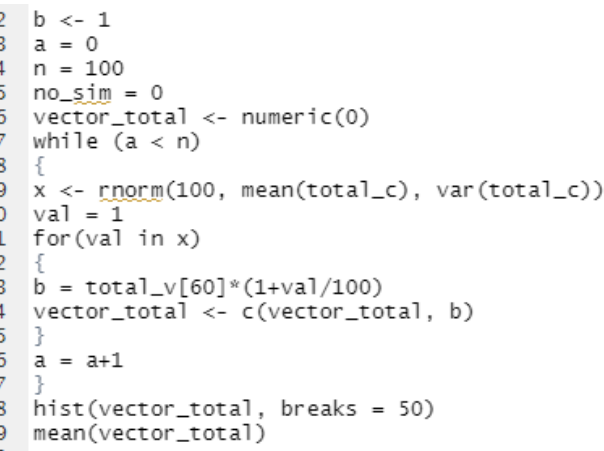

Figure 3: The GVA Model

Source: Own code

This particular code simulates the total GVA. To simulate each sector, multiple variables and vectors have been created and changed for each specific case.

\subsection{The European Model}

The previous two models assume that an economy will develop based on historical evolution. This is most definitely not the case, as business conditions change and so does the total output. Moreover, some industries could perform worse as an economy evolves 
while others could perform better. In this respect, the new behavior comes from a distribution that is shared with all other EU27 countries.

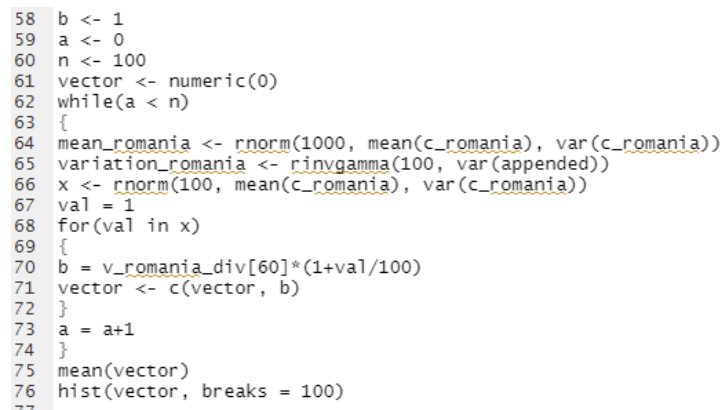

Figure 3: The European Model

Source: Own code

To do so, a vector was created in which all marginal GDP changes were appended so that a distribution could be simulated based on their mean and variance. Thus, in these simulations, Romania's distribution mean is fit to a normal distribution, while the country's variation is fit to an inverse gamma distribution with the variation of EU27 countries.

\section{Model Testing}

To ensure the aforementioned models produce adequate results, the models have been tested with a small number of simulations on already existing GDP values. 2020 Q4 was chosen as the reference year for these tests while the number of simulations was chosen to be 100 sets of 100 simulations. Furthermore, the simulated GDP value was chosen as the mean of the total simulations. Thus, for 2020 Q4, the historical model, which is solely based on historical evolution, produces a mean GDP of EUR 57.8 billion, the total GVA model, ,which is similar to the historical model, produces a simulated mean of EUR 52.9 billion and taxes less subsidies of EUR 5.4 billion resulting in a total GDP of EUR 58.3 billion. The GVA approach by simulating all sectors produces a GDP value of EUR 57.9 billion while the European model produces a mean GDP of EUR 57.7 billion. The table below presents the test results against the actual GDP value, in EUR billion at current prices, which was taken from Eurostat (2021).

Table 1: Testing Results

\begin{tabular}{|r|c|r|r|r|}
\hline $\begin{array}{r}\text { Actual } \\
\text { GDP 2020 Q4 }\end{array}$ & $\begin{array}{r}\text { Historical } \\
\text { model }\end{array}$ & $\begin{array}{r}\text { GVA } \\
\text { (total) }\end{array}$ & $\begin{array}{r}\text { GVA } \\
\text { (by sectors) }\end{array}$ & $\begin{array}{c}\text { European } \\
\text { Model }\end{array}$ \\
\hline 57.5 & 57.8 & 58.3 & 57.9 & 57.7 \\
\hline
\end{tabular}

Source: Eurostat, authors'calculation

The tests were considered adequate for implementation since the simulations produce values that are marginal to the actual value. A margin of error has also been assumed to be acceptable since forecasts and simulations are unlikely to produce a perfect value. In terms of simulations on forward looking GDP, it has been decided that to achieve model convergence, and considering that the more simulations are running, the closer the mean could be to the real value, at least one million simulations should be run per model. One more consideration that needs to be addressed with respect is that the GDP values have 
been divided by 1000 due to efficiency matters such as computing time and to avoid a potential data overflow.

\section{Results}

In order to check that the algorithm is working without a data overflow, a set of instructions was added that prints the number of simulations that the algorithm executed for every $10 \%$ completion. This additional set of instructions decreases the efficiency by increasing the running time, however, it ensures that the algorithm does not fail, and it does not enter in an infinite repetitive loop due to an overflow. After one million simulations using the historical model, the mean GDP value produced is EUR 58.9 billion for 2021 Q1, while GVA methodology produced a simulated GDP of EUR 58.6 billion. Last but not least, the European model produced a mean GDP of EUR 58.4 billion. Thus, after running the models several times with one million simulations each time, the mean value is EUR 58.6 billion for Q1 2021. The image below is a graphical representation of the total simulated values for the European Model.

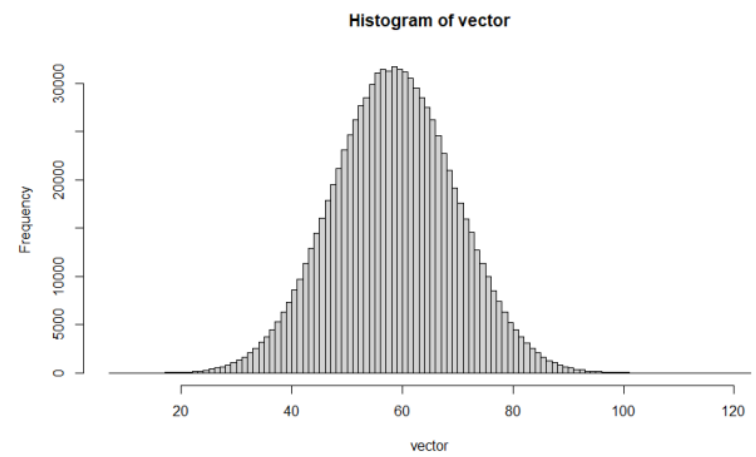

Figure 4: Simulation Results

Source: Simulations in $\mathrm{R}$ based on own code

On the other hand, simulated values can be produced for the year 2021 as well. This was done by simulating each quarter and them summing the values up. Thus, as a result of these simulations, Romania's GDP could reach a total value of EUR 224 billion for 2021, implying a $3.2 \%$ upside for the whole year. The chart below shows a graphical representation of some of the simulated 2021 outcomes.

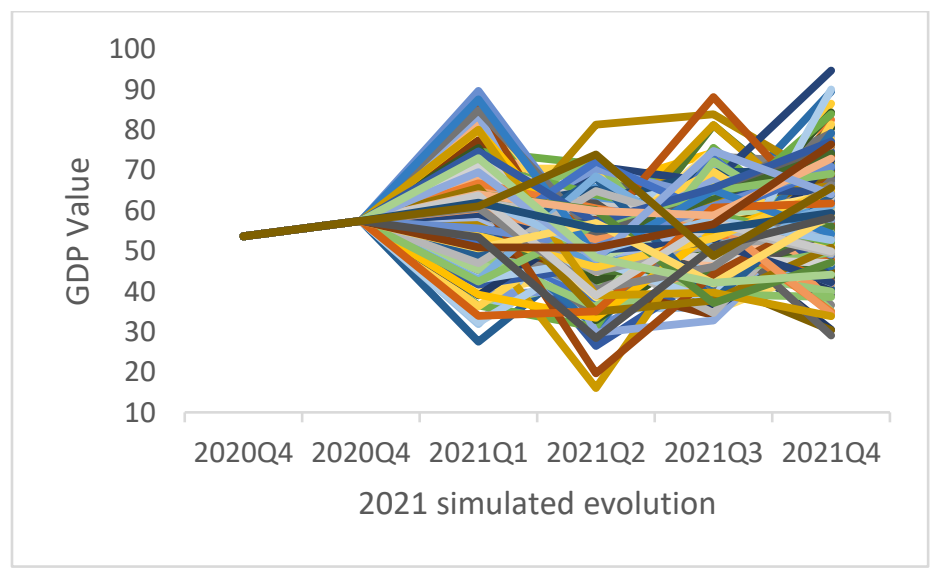


Figure 5: Simulation Results

Source: Simulations in $\mathrm{R}$ based on own code

Table 2: Means of the simulated quarterly values in EUR

\begin{tabular}{|r|r|r|r|}
\hline 2021 & 2021 & 2021 & 2021 \\
\hline 58.4 & 51.1 & 55.3 & 59.4 \\
\hline
\end{tabular}

Source: authors'calculation

\section{Discussion}

As stated, this paper has aimed to simulate the Romanian quarterly GDP data to estimate what the country's economy could look like in 2021 with the aim of creating a perspective on the long-term growth potential. In order to do so, two main research questions have been asked. First, how the global health crisis could project itself on Romania's GDP in 2021. Second, to determine to what extent can Romania face its current challenges with increased governmental expenditure and to determine the possible solutions that the country's economic framework could come up with to create a sustainable system going forward.

Thus, to get a sense of what the simulated values could imply, the aforementioned results have been compared to the already existing institutional forecasts for 2021 . According to the European Commission (2021), the country could grow by $3.8 \%$ in 2021 . The International Monetary Fund (2021) is bullish in their estimations with a projected $6 \%$ growth at constant prices, the World Bank (2021) sees a growth of $4.3 \%$ while the Romanian National Commission for Strategy and Forecasting (2021) projects a growth of $4.3 \%$. The simulations in this paper produced a mean growth of $3.2 \%$, which is more conservative than all other forecasts mentioned in this paper.

As such, the global health crisis, which currently affects not only Romania, but the whole world in 2021 as well, through various measures to combat the virus spread, seems to project its impact on the economy. Based on this fact, but not exclusively, Romania's budgetary pressures are likely to remain at concerning levels throughout 2021 and going forward. In fact, based on the simulation model, the budget deficit could come down to approximately negative $7.8 \%$ while debt relative to GDP could go up to $49 \%$. Furthermore, consumption could fractionally increase by $4.2 \%$. Looking at the GVA computation methodology, the best performing sector by growth rate could be communications which could be mainly driven by a substantial growth in information technology. With regards to the first research question, it becomes clear that the ongoing health crisis still has negative implications for 2021 and going forward, as the budget balance will not achieve minimum accepted levels by the European Union. Furthermore, this could also imply economic pressures due to the potential sanctions that the country may be eligible for.

Since Romania had already been facing these pressures before, several solutions are hence proposed in this paper. These solutions may aid in policymaking; however, this paper's objective has not been to analyze the potential impact of the proposed solutions, but to use the simulated outcomes to find the respective solutions. The potential impact of the proposed solutions could therefore be a continuation of this research. Considering the existing situation and acknowledging the fact that the full impact of the sanitary crisis is yet unknown, one approach towards ensuring a sustainable economic system going forwards is to make use of the expected revenues while also taking advantage of the relative low interest rates in EUR denominated instruments, but only to a level that does not significantly impact the respective currency. Even though this approach has been widely used to support affected sectors, it can still be used without causing major disruptions, albeit on a marginally lower scale, where less debt instruments would be issued as the economy gradually rebounds. Another potential solution is to increase the total tax collection, not by increasing the actual 
taxable income but by combating fraud while also temporarily exempting small and medium businesses from a portion of their tax obligations throughout the economy for a very short while, and possibly only after the gradual recovery starts. This would affect tax revenues, but it may aid in the medium towards long-term as it could mean businesses would have additional resources to trade. This could also imply higher inflationary pressures; however, an estimation of this potential impact could be a topic for future research. Last but not least, another proposed solution is to increase the EU cohesion funds absorption as the country's absorption inflows currently stand at around $10 \%$ per year according to the European Commission (2021). A faster absorption rate could translate not only into a faster rebound but could also open a path for sustainable business models to emerge.

\section{Conclusion}

The objective of this paper has been to run probabilistic simulations on Romania's quarterly GDP to estimate what the economic growth could look like in 2021 and address the questions of how the impact of the global health crisis could impact Romania both in 2021 and going forward, and to what extent could Romania face its current budgetary pressures. The methodology used in this paper was to code an algorithm that can run simulations using the $\mathrm{R}$ software package. The main tradeoff in running such simulations is the time needed to run them due to exponential complexity. Enough simulations were needed for model convergence. As such, it has been decided that at least one million simulations would achieve this goal.

This paper mainly finds that Romania's GDP could grow by a mean of $3.2 \%$ in 2021 in nominal terms, that private consumption could increase by $4.2 \%$, that the budget balance is likely to stay in negative territory by $7.8 \%$ and the highest growing sector could be communications, especially information technology. The proposed solutions are to keep taking advantage of the low interest rates, but only up to a level that does not impact the respective currency, to reduce fraud and thereby increase tax collection while also supporting small and medium businesses through very short-term tax exemptions which could be beneficial in the long-run, and to increase the EU cohesion funds absorption rates as Romania currently manages to absorb approximately $10 \%$ of the allocated funds per year. The purpose of this paper has not been to estimate what the potential economic impact of these solutions could be. This may be subject to future research.

Nevertheless, the whole world is faced with unprecedented times in recent history. These events have had a significant impact on society and therefore on the economy. In such times, it may be difficult to understand the full impact of these events as they have yet to completely end. In such times, it is up to us to find cohesion and understanding, as the opposite might not only affect some on an individual level, it would alter the fabric of the society as a whole, and it might lead us towards a path that is bound to gloom.

\section{Research Limitations}

This paper is limited to the known facts and factors that may disrupt the Romanian economic growth. This paper acknowledges that there may be a large number of other factors that may cause growth impediments, and which are not known as of the writing of this paper. Besides, this research is limited to relatively low computing power, and the model cannot pinpoint the exact moment in time when a disruption may even take place. Thus, future research is imperative to better understand the events that are currently going on as well as their full implications. 


\section{Acknowledgements}

The publication of this article was supported by the 2020 Development Fund of the Babeș-Bolyai University.

\section{References}

1. Anon., n.d. European Commission. [Online].

2.Bank, W., 2021. Country Context. [Online].

3.Bank, W., 2021. World Bank. [Online].

4.Commission, E., 2021. EC. [Online].

5.Daianu, D. \& Kallai, E., 2008. Disinflation and Inflation Targeting in Romania. Romanian Journal of Economic Forecasting, Volume 1.

6.European Commission, 2021. European Structural and Investment Funds. [Online].

Eurostat, 2021. Eurostat. [Online].

7.Eurostat, 2021. Eurostat. [Online].

8.Geman, S. \& Geman, D., 1984. Stochastic Relaxation, Gibbs Distributions, and the 9.Bayesian Restoration of Images. IEEE Transactions on Pattern Analysis and Machine Intelligence, 6(6), pp. 721741.

10.Glocker, C. \& Wegmuller, P., 2017. Business Cycle Dating and Forecasting with Real-time Swiss GDP Data, s.I.: Austrian Institute for Economic Research.

11.Gorka, J., Osinska, M. \& Stawicki, J., 2002. Modelling and Forecasting the GDP Structure of Polish and Estonian Economies in Transition Period Using Markov Chains. Contributions to Econometrics.

12. Hastings, W., 1970. Monte Carlo sampling methods using Markov chains and their applications.

Biometrika, 57(1), p. 97.

International Monetary Fund, 2021. World Economic Outlook Database. [Online].

13.Kluza, K., 2016. Sustainability of local government sector debt. Evidence form Monte-Carlo simulations. Journal of Local Self-Government, 14(1), pp. 115-132.

14.Kruger, F., Lerch, S. \& Thorarinsdottir, T., 2017. Probabilistic Forecasting , s.I.: Heidelberg University.

15.Landefeld, S., 2008. Taking the Pulse of the Economy: Measuring GDP. Journal of Economic Perspectives, 22(2), pp. 193-216.

16.Lerche, I. \& Mudford, B., 2005. How Many Monte Carlo Simulations Does one Need to do?. Energy Exploration \& Exploitation, 23(6), pp. 405-427.

17.Little, R., 2006. The American Statistician, 60(3).

18.Metropolis, N., 1953. Equation of State Calculations by Fast Computing Machines, Los Alamos: Los Alamos Scientific Laboratory.

19.Olerichs, $\quad$ C., $2017 . \quad$ Medium. [Online] Available at: https://medium.com/@c.oelrichs/simple-bayesian-models-of-economic-growtheb9251ad4d55

20.Pitt, M., Silva, R., Giordani, P. \& Kohn, R., 2020. On some properties of Markov chain Monte Carlo simulation methods. Journal of Econometrics, 171(2), pp. 134-151.

21.Romanian Commission for Strategy and Forecasting, 2021. Projection of the main macroeconomic indicators. [Online].

22.Roy, V., 2020. Convergence diagnostics for Markov chain Monte Carlo, s.l.: lowa State University. 23.Savoiu, G., Burtescy, E. \& Dinu, V., 2017. A Monte Carlo method simulation of the European funds that can be accessed by Romania in 2014-2020. E a M: Ekonomie a Management, 20(3), pp. 19-35. 24.Simionescu, M., 2020. Bayesian combined forecasts and Monte Carlo simulations to improve inflation rate predictions in Romania. Research Papers in Economics and Finance, 4(1).

25.Simionescu, M., 2020. Bayesian combined forecasts and Monte Carlo simulations to improve inflation rate predictions in Romania. Research Papers in Economics and Finance, 4(1).

26.Svensson, L., 2010. Inflation Targeting, Cambridge, Massachusets: National Bureau of Economic Research.

27.Tanner, M. \& Wong, W., 1987. The Calculation of Posterior Distributions by Data Augmentation. Journal of the American Statistical Association, 82(398), pp. 528-540.

28.Timu, $2021 . \quad$ Bloomberg. [Online] Available at: https://www.bloomberg.com/news/articles/2021-02-10/romanian-premier-says-2021draft-budget-gap-is-still-high 
The Annals of the University of Oradea. Economic Sciences

TOM XXX, $1^{\text {st }}$ Issue, July 2021

29.Wolf, L., Anselin, L. \& Arribas-Bel, D., 2017. Stochastic Efficiency of Bayesian MCMC in Spatial Econometric, s.l.: Regional Science Association International. 